\title{
Eficacia de la infiltración periarticular con anestésico local y adyuvantes para control del dolor postquirúrgico en la artroplastía total de rodilla
}

\author{
Effectiveness of peri-articular infiltration with local anesthetic and \\ adjuvants for post-surgical pain control in total knee replacement
}

\author{
Campos-Flores D,* Malpica-Ramírez LM, ${ }^{\ddagger}$ Cariño-Cepeda C, ${ }^{\ddagger}$ \\ Fernández de Lara-Castilla LG, ${ }^{\ddagger}$ Gálvez-Romero JL ${ }^{\S}$
}

Hospital Regional Puebla, ISSSTE.

RESUMEN. Introducción: La artroplastía total de rodilla (ATR) es uno de los tratamientos ortopédicos más exitosos; sin embargo, se ha asociado a dolor postquirúrgico intenso en $30-60 \%$ de los pacientes. Nosotros planteamos que la infiltración de la cápsula articular de la rodilla durante la cirugía disminuirá el dolor postquirúrgico. Material y métodos: Estudio experimental, aleatorio, doble ciego, en pacientes sometidos a ATR unilateral entre Abril de 2018 a Enero de 2019. Los pacientes fueron divididos en dos grupos, el primero infiltración con placebo y el segundo con solución anestésica y adyuvantes (fentanilo, epinefrina y ketorolaco). Se cuantificó mediante escala visual análoga (EVA) del dolor a las cuatro, seis, ocho, 12, 18, 24, 36 y 48 horas postquirúrgicas, así como del consumo de analgésicos opioides y antieméticos. Resultados: Veinte pacientes en cada grupo, con un seguimiento de cuatro semanas. No hubo diferencias significativas en las características demográficas entre ambos grupos. Se observó un mejor control del dolor postquirúrgico en el grupo que recibió infiltración con anestésico y adyuvante, además de una disminución en el consumo de analgésicos opioides y antieméticos. No hubo diferencia en sangrado ni
ABSTRACT. Introduction: Total knee arthroplasty (TKA) is one of the most successful orthopedic treatments, however, it has been associated with severe postsurgical pain in $30-60 \%$ of patients. We propose that infiltration of the articular capsule of the knee during surgery will decrease postsurgical pain. Material and methods: Experimental, randomized, double-blind study in patients undergoing unilateral TKA between April 2018 and January 2019. Patients were divided into two groups, the first infiltration with placebo and the second with anesthetic solution and adjuvants (fentanyl, epinephrine and ketorolac). Pain was measured with the visual analog scale (VAS) at 4, 6, 8, $12,18,24,36$ and 48 hours postsurgical, as well as the consumption of opioid analgesics and antiemetics. Results: 20 patients in each group, with a follow-up of 4 weeks. There were no significant differences in demographic characteristics between the two groups. Better control of postsurgical pain was observed in the group that received infiltration with anesthetic and adjuvant, as well as a decrease in the consumption of opioid analgesics and antiemetics. There was no difference in bleeding or

\section{Nivel de evidencia: I}

* Médico residente Traumatología y Ortopedia.

‡ Médico adscrito al Servicio de Traumatología y Ortopedia.

$\S$ Jefe Departamento de Investigación.

Instituto de Seguridad y Servicios Sociales de los Trabajadores del Estado (ISSSTE), Hospital Regional Puebla.

Correspondencia:

Dr. David Campos-Flores

2 Poniente 214, Col. Centro, C.P. 75700, Tehuacán, Puebla.

E-mail: campos.tyo@gmail.com

Recibido: 09-06-2020. Aceptado: 15-01-2021.

Citar como: Campos-Flores D, Malpica-Ramírez LM, Cariño-Cepeda C, Fernández de Lara-Castilla LG, Gálvez-Romero JL. Eficacia de la infiltración periarticular con anestésico local y adyuvantes para control del dolor postquirúrgico en la artroplastía total de rodilla. Acta Ortop Mex. 2021; 35(2): 169-173. https://dx.doi.org/10.35366/101861

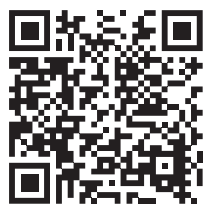


en la incidencia de infecciones entre ambos grupos. Conclusión: La infiltración pericapsular es un método seguro y eficaz, como parte de la analgesia multimodal en la artroplastía total de rodilla, ya que disminuye el dolor postquirúrgico, el consumo de opioides y antieméticos y no incrementa el sangrado postquirúrgico.

Palabras clave: Dolor, infiltración, rodilla, cápsula articular, prótesis.

\section{Introducción}

La artroplastía total de rodilla (ATR) se ha realizado rutinariamente durante más de 40 años como parte del manejo de gonartrosis severa. Su éxito se basa en la capacidad de mejorar la calidad de vida del paciente, reduciendo el dolor y la función a largo plazo; sin embargo, $40 \%$ de los pacientes se quejan de los resultados obtenidos. ${ }^{1}$

El dolor postoperatorio intenso se presenta en 30 a $60 \%$ de los casos, ${ }^{2}$ por lo que el control del dolor postquirúrgico se ha convertido en una gran preocupación tanto para el paciente como para el cirujano. Un pobre control del dolor postquirúrgico tendrá efectos adversos sobre la movilización temprana, aumentando la incidencia de complicaciones asociadas además de resultados funcionales poco satisfactorios. ${ }^{3}$

En un intento por controlar el dolor postquirúrgico, se ha desarrollado el manejo multimodal del dolor, el cual incluye manejo con antiinflamatorios no esteroideos orales o intravenosos, analgésicos narcóticos, anestesia epidural continua, analgesia controlada por el paciente, bloqueo de nervios periféricos y la infiltración periarticular. ${ }^{4,5}$ Todas estas medidas son reconocidas como métodos efectivos para el control del dolor postquirúrgico. ${ }^{6,7}$

El bloqueo de nervios periféricos, como parte de la analgesia multimodal, se ha asociado a varios efectos adversos, entre los que destacan el riesgo de caída, lesión nerviosa y pérdida temporal del control motor, provocando un retardo en la movilización, ${ }^{8,9,10}$ aumentando el riesgo de complicaciones como la trombosis venosa profunda.

Los medicamentos opioides son los fármacos más potentes para el manejo del dolor; sin embargo, se asocian a varios efectos secundarios, entre los que destacan: depresión ventilatoria, sedación, náuseas en el período postquirúrgico, prurito, retención urinaria e íleo, ${ }^{11}$ lo cual prolonga la estancia hospitalaria y los costos de la misma. La infiltración local de medicamentos opioides permite una mayor concentración local de la droga, disminuye los niveles sistémicos del fármaco, reduciendo el riesgo de efectos adversos. ${ }^{12,13}$

La infiltración pericapsular para el manejo del dolor postquirúrgico es una intervención técnicamente de menor demanda, comparada con la infiltración de nervios periféricos, reduce el consumo de opioides con menores efectos secundarios y mejora la movilización en el período postquirúrgico, permitiendo un mejor resultado a mediano plazo. ${ }^{14}$ in the incidence of infections between the two groups. Conclusion: Peri-capsular infiltration is a safe and effective method, as part of multimodal analgesia in total knee arthroplasty, as it decreases postsurgical pain, opioid and antiemetic use and does not increase postsurgical bleeding.

Keywords: Pain, infiltration, knee, joint capsule, prosthesis.

Las distintas fórmulas para infiltración pericapsular incluyen anestésicos locales junto con la adición de varias drogas, como son antiinflamatorios no esteroides y epinefrina. Pueden contener también antibióticos, esteroides y otros agentes localmente activos. Estos cocteles han mostrado ser efectivos para el dolor postquirúrgico, pero tienen un tiempo de acción corto. El coctel tradicional incluye anestésicos locales como son bupivacaína o ropivacaína, que han sido la base de los mismos. ${ }^{15}$ En este estudio se formuló un coctel con base en los medicamentos disponibles dentro del cuadro básico de medicamentos para nuestra unidad hospitalaria.

El objetivo del estudio es valorar la eficacia de la infiltración pericapsular con anestésico local y adyuvante, para control del dolor postquirúrgico en la artroplastía total de rodilla.

\section{Material y métodos}

Protocolo de investigación con número de registro 102.2018 en la dirección de investigación del ISSSTE, es un estudio experimental, comparativo, longitudinal y prospectivo de Abril 2018 a Enero 2019. Se realizó un muestreo probabilístico aleatorio simple, con doble ciego y placebo control.

Con base en un estudio previo para la diminución del dolor postoperatorio con infiltración pericapsular realizado por Song y colaboradores, se encuentra con una desviación estándar de 2, si la diferencia real entre el promedio del grupo experimental y grupo control es de 1.9 , se requiere un total de 20 pacientes por cada grupo, con la intención de rechazar la hipótesis nula, con una potencia de $80 \%$ y con una probabilidad de error alpha de 0.05 .

Criterios de inclusión: pacientes sometidos a artroplastía total de rodilla unilateral en el período del estudio, edad entre 18-85 años.

Criterios de exclusión: alergia a alguno de los medicamentos usados en el coctel para infiltración pericapsular, enfermedad renal crónica o enfermedad hepática, osteotomías en la rodilla afectada, condiciones neurológicas o sicológicas que impidan el llenado de los formatos de recolección de datos, patologías de columna vertebral que impidan la realización de anestesia raquídea, presencia de diabetes mellitus con sospecha o diagnóstico de neuropatía periférica. 
Criterios de eliminación: pacientes que decidan abandonar el protocolo de estudio, que presenten reacciones adversas a alguno de los medicamentos, con mala evolución clínica postquirúrgica que impida la continuación del protocolo, complicaciones asociadas con la anestesia raquídea.

El protocolo prequirúrgico fue el mismo. Bajo anestesia espinal, con uso de isquemia, se realizó abordaje tipo Insall trivectorial para implantación de prótesis de rodilla cementada postero y no postero-estabilizada Genesis II Smith \& Nephew $^{\circledR}$ después de colocado el implante (Figura 1), se infiltró una de dos posibles soluciones: a) $100 \mathrm{mg}$ bupivacaína, $100 \mu \mathrm{g}$ fentanilo, $0.5 \mathrm{mg}$ epinefrina $1: 1,000$ y $30 \mathrm{mg}$ ketorolaco, diluido con solución salina isotónica $0.9 \%$, llevando la mezcla total a $50 \mathrm{ml}$; y b) solución salina isotónica $0.9 \% 50 \mathrm{ml}$.

Se dividió la solución en cinco partes de $10 \mathrm{ml}$ cada una, se infiltró:

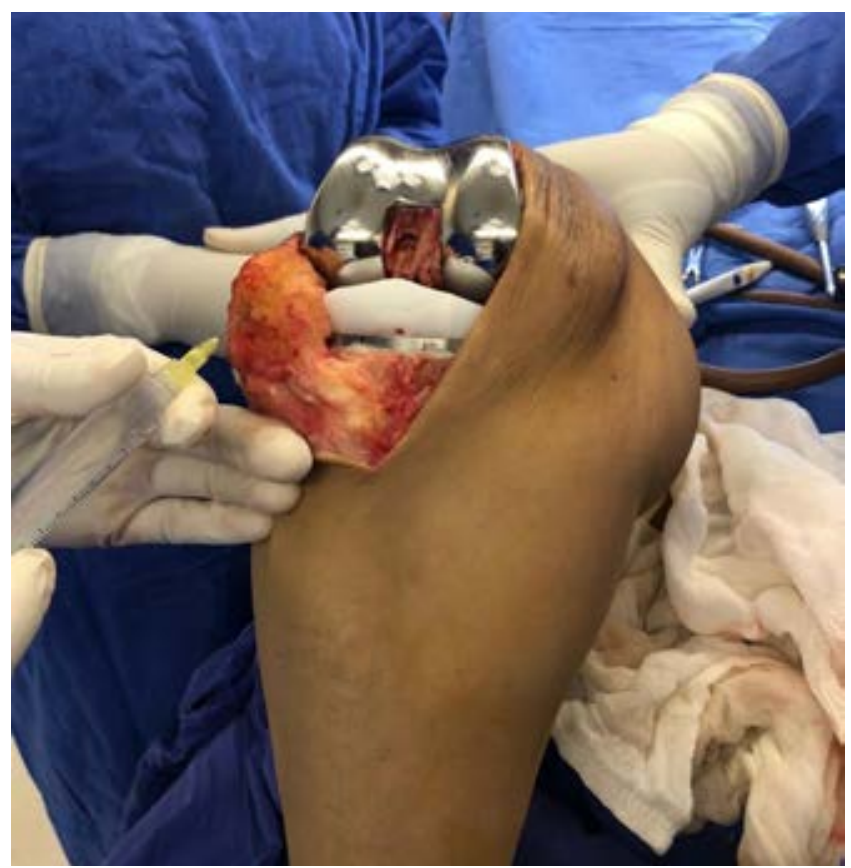

Figura 1: Técnica de infiltración periarticular.
1. Cápsula lateral.

2. Cápsula medial.

3. Tendón rotuliano.

4. Tendón cuadricipital.

5. Tendones de pata de ganso.

Después se cerró por planos con sutura absorbible, piel con sutura no absorbible y se colocó un drenaje por 24 horas.

Se calificó el dolor con la escala visual análoga (EVA) en reposo a las cuatro, seis, ocho, 12, 18, 24, 36 y 48 horas postquirúrgicas. Se tomó citometría hemática de control a las 24 horas postquirúrgicas, valorando la disminución del porcentaje de hematocrito. Se midió el consumo de analgésicos opioides, así como el consumo de medicamentos antieméticos en ambos grupos durante la estancia hospitalaria.

El análisis de datos se recolectó mediante el programa Excel, las variables numéricas se describieron con media y desviación estándar; las variables nominales se describieron en frecuencia y porcentaje. Para la inferencia estadística se empleó la t de Student para diferencia de las medias en grupos independientes o bien U de Mann Whitney (si se comporta como una distribución no normal), a través de programa SPSS versión 21.

\section{Resultados}

Se incluyeron 40 pacientes, asignados en dos grupos: a) infiltración con solución anestésica; y b) grupo placebo. Ambos grupos presentaron características demográficas homogéneas (Tabla 1).

La escala visual análoga del dolor (EVA) a las cuatro, ocho, 12, 16, 32 y 48 horas postquirúrgicas mostró una diferencia significativa $(\mathrm{p}<0.05)$ en todas las mediciones $(\mathrm{Ta}-$ bla 2), a favor del grupo que recibió infiltración con anestésico a lo largo de todo el seguimiento (Figura 2).

También mostró una disminución en el consumo de analgésicos opioides y antieméticos, esto en contraste con el grupo placebo $(\mathrm{p}<0.05)$, con una dosis acumulativa de Tramadol, promedio de $220 \mathrm{mg}$ en el grupo placebo y 10 mg en el grupo experimental (Tabla 3). No hubo diferencia significativa en la disminución del porcentaje de hemato-

\begin{tabular}{|c|c|c|c|c|c|}
\hline Variable & Placebo, n (\%) & Anestésico, n (\%) & Placebo & Anestésico & $\mathrm{p}$ \\
\hline Pacientes & $20(100)$ & $20(100)$ & & & \\
\hline Edad, años & & & $68.1 \pm 8.9$ & $68.8 \pm 8.3$ & 0.79 \\
\hline \multicolumn{6}{|l|}{ Género } \\
\hline Hombre & $9(45)$ & $9(45)$ & & & 0.62 \\
\hline Mujer & $11(55)$ & $11(55)$ & & & \\
\hline IMC promedio & & & $29.16 \pm 3.32$ & $28.77 \pm 4.28$ & 0.75 \\
\hline Hipertensión arterial sistémica & $9(45)$ & $9(45)$ & & & 0.62 \\
\hline Días de hospitalización & & & $2.05 \pm 0.22$ & $2.25 \pm 0.55$ & 0.14 \\
\hline
\end{tabular}


Tabla 2: Escala visual análoga postquirúrgica en grupo placebo y grupo de estudio.

\begin{tabular}{|c|c|c|c|}
\hline $\begin{array}{c}\text { Variable } \\
\text { (EVA) }\end{array}$ & Placebo & Estudio & $\mathrm{p}^{*}$ \\
\hline 4 & $7.25 \pm 2.63$ & $4.15 \pm 2.66$ & 0.001 \\
\hline 6 & $7.35 \pm 2.66$ & $4.40 \pm 2.58$ & 0.001 \\
\hline 8 & $6.65 \pm 2.03$ & $3.50 \pm 2.48$ & 0.001 \\
\hline 12 & $6.65 \pm 1.76$ & $3.25 \pm 2.61$ & 0.001 \\
\hline 18 & $6.65 \pm 1.95$ & $4.00 \pm 1.81$ & 0.001 \\
\hline 36 & $6.65 \pm 1.84$ & $4.05 \pm 1.85$ & 0.001 \\
\hline 24 & $6.05 \pm 1.96$ & $3.80 \pm 1.47$ & 0.001 \\
\hline 48 & $5.75 \pm 2.02$ & $3.60 \pm 1.76$ & 0.001 \\
\hline $\begin{array}{l}* \text { Diferencia } \\
\text { estadística de } \\
\text { EVA = escala }\end{array}$ & $\begin{array}{l}\text { se obtuvo con } \\
\text { áloga. }\end{array}$ & nt con una sig & \\
\hline
\end{tabular}

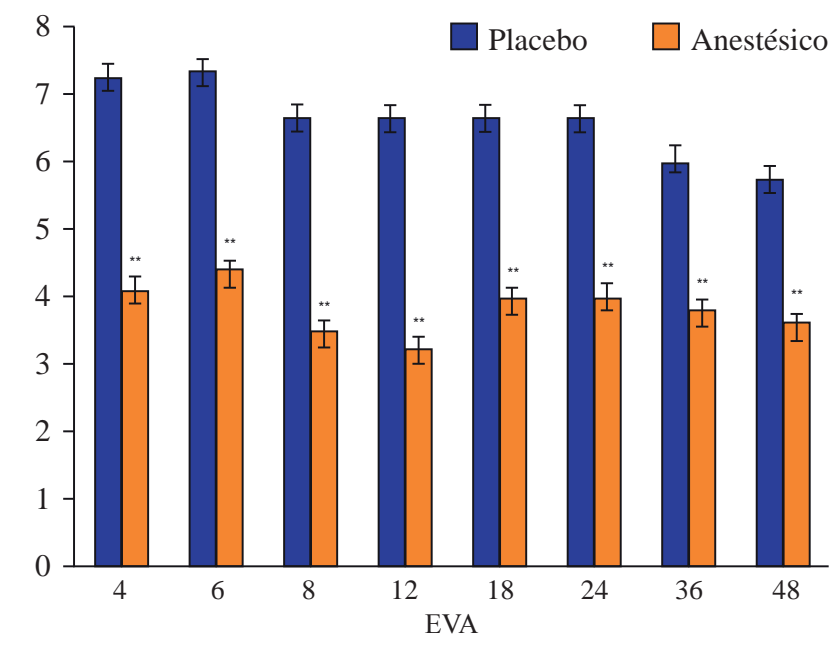

Figura 2: Dolor postquirúrgico medido con escala visual análoga en pacientes sometidos a artroplastía total de rodilla.

** La diferencia de promedios se analizó a través de ANOVA, se obtuvo una $\mathrm{p}=0.001$.

crito entre los grupos ( $p=0.42)$, así como tampoco hubo diferencia en los días de estancia hospitalaria entre los dos grupos $(\mathrm{p}=0.14)$.

\section{Discusión}

En nuestro estudio, se observa que la infiltración pericapsular con anestésico local y adyuvantes es un método eficaz para el control del dolor postquirúrgico en los pacientes sometidos a ATR. Diversos estudios con características metodológicas similares concuerdan con nuestros resultados, esto también se observa con diferentes técnicas de infiltración y con diferentes mezclas de formulación. 12,15,16,17

Gibbins y colaboradores ${ }^{12}$ analizaron diversas formulaciones compuestas por anestésicos locales y adyuvantes utilizados en 12 estudios, todos ellos compuestos por anestésicos locales (150 a $300 \mathrm{mg}$ de ropivacaína y de 50-100 mg de bupivacaína) y adyuvantes (30 mg ketorolaco, $0.5 \mathrm{mg}$ adrenalina, 5 a $10 \mathrm{mg}$ morfina, $100 \mu \mathrm{g}$ de fentanilo). Desta- ca que, independientemente de la composición de cada una de las posibles soluciones analgésicas con adyuvantes, todas demostraron una disminución de los valores de la EVA del dolor en los pacientes sometidos a ATR.

En cuanto al sangrado postquirúrgico, Lombardi y su equipo $^{15}$ demostraron menor sangrado en los pacientes sometidos a infiltración periarticular, contrario a los resultados obtenidos en nuestro estudio; sin embargo, otros autores coinciden con nuestros resultados al no encontrar diferencias en el sangrado entre el grupo experimental y el grupo placebo, lo cual sugiere que nuestra técnica no incrementa la probabilidad de sangrado más allá de lo convencionalmente esperado por las características del procedimiento quirúrgico.

Pocos estudios describen con detalle la técnica de infiltración, nuestro estudio empleó la técnica descrita por Guild GN y colaboradores ${ }^{17}$ partiendo de la neuroanatomía de la rodilla, se describen las áreas de mayor sensibilidad y por lo tanto las que requieren ser infiltradas con anestésico local, esto al evitar la aleatorización de la infiltración, fortalece la reproducibilidad de los resultados obtenidos. Además, si no se infiltra la cápsula posterior, se disminuye el riesgo de complicaciones neurovasculares y se mejora el control del dolor postquirúrgico, ${ }^{7}$ por lo que nuestra técnica de infiltración es segura al no tener evidencia de lesión neurológica o vascular en ninguno de los participantes del estudio.

Terkawi y su equipo ${ }^{10}$ observaron un menor consumo de medicamentos opioides en los pacientes sometidos a infiltración intraarticular con anestésico local y adyuvantes, concordando con los resultados obtenidos en nuestro estudio, se demuestra que la infiltración periarticular es un método eficaz para disminuir el consumo de este tipo de medicamentos. Este mismo estudio reportó una incidencia de 2\% de infecciones asociadas a infiltración periarticular, aunque este porcentaje coincide con el riesgo de infección asociado con el procedimiento quirúrgico en sí, ${ }^{18}$ por lo que es probable que la infiltración periarticular no aumente la incidencia de infecciones.

La infiltración periarticular supone una técnica sencilla, ${ }^{19}$ lo que facilita la reproducibilidad de los resultados obtenidos en este estudio.

Dentro de las limitaciones del estudio se encuentra el seguimiento corto de pacientes, que sólo abarcó hasta las 48

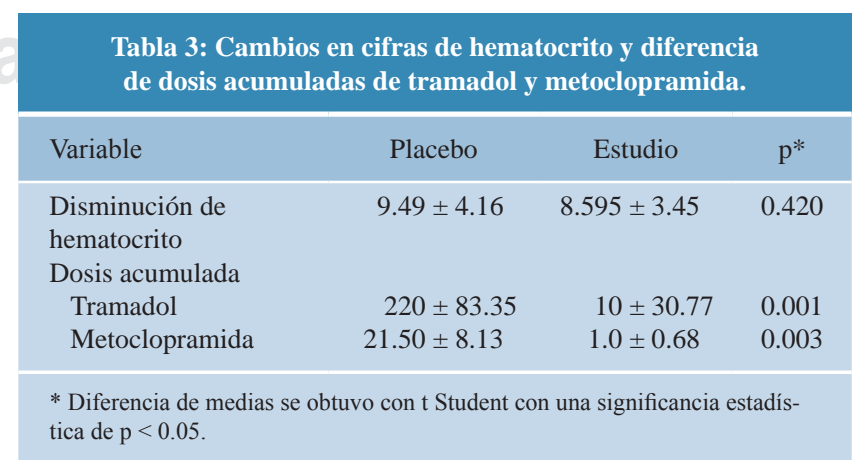


horas postquirúrgicas, la falta de una evaluación funcional inmediata y el comparar de manera objetiva otras modalidades de analgesia; es necesaria una muestra más amplia para valorar el efecto global de la infiltración periarticular.

\section{Conclusión}

La infiltración periarticular es un método seguro y eficaz, como parte de la analgesia multimodal en la artroplastía total de rodilla, disminuye el consumo de opioides y antieméticos contra placebo y no incrementa el sangrado postquirúrgico.

\section{Referencias}

1. Price AJ, Alvand A, Troelsen A, Katz JN, Hooper G, Gray A, et al. Knee replacement. Lancet [Internet]. 2018; 392(10158): 1672-82. Available from: http://dx.doi.org/10.1016/S0140-6736(18)32344-4

2. Li J wen, Ma Y shuo, Xiao L kun. Postoperative pain management in total knee arthroplasty. Orthop Surg. 2019; 11(5): 755-61.

3. Melhuish TM, White LD. Peri-operative strategies to reduce pain post total knee arthroplasty. J Pain Relief. 2016; 05(04).

4. Song MH, Kim BH, Ahn SJ, Yoo SH, Kang SW, Kim YJ, et al. Periarticular injections of local anaesthesia can replace patient-controlled analgesia after total knee arthroplasty: a randomised controlled study. Int Orthop. 2016; 40(2): 295-9.

5. Seangleulur A, Vanasbodeekul P, Prapaitrakool S, Worathongchai S, Anothaisintawee T, McEvoy M, et al. The efficacy of local infiltration analgesia in the early postoperative period after total knee arthroplasty: a systematic review and meta-analysis. Eur J Anaesthesiol. 2016; 33(11): 816-31.

6. Motififard M, Omidian A, Badiei S. Pre-emptive injection of periarticular-multimodal drug for post-operative pain management in total knee arthroplasty: a double-blind randomized clinical trial. Int Orthop [Internet]. 2017; 41(5): 939-47. Available from: http://dx.doi. org/10.1007/s00264-016-3357-2

7. Pinsornsak P, Nangnual S, Boontanapibul K. Multimodal infiltration of local anaesthetic in total knee arthroplasty; is posterior capsular infiltration worth the risk? Bone Joint J. 2017; 99B(4): 483-8.

8. Fenten MGE, Bakker SMK, Touw DJ, van den Bemt BJF, Scheffer GJ, Heesterbeek PJC, et al. Pharmacokinetics of $400 \mathrm{mg}$ ropivacaine after periarticular local infiltration analgesia for total knee arthroplasty. Acta Anaesthesiol Scand. 2017; 61(3): 338-45.

9. Yan H, Cang J, Xue Z, Lu J, Wang H. Comparison of local infiltration and epidural analgesia for postoperative pain control in total knee arthroplasty and total hip arthroplasty: a systematic review and metaanalysis. Bosn J basic Med Sci. 2016; 16(4): 239-46.

10. Terkawi AS, Mavridis D, Sessler DI, Nunemaker MS, Doais KS, Terkawi RS, et al. Pain management modalities after total knee arthroplasty. Anesthesiology. 2017; 126(5): 923-37.

11. Sakamoto B, Keiser S, Meldrum R, Harker G, Freese A. Efficacy of liposomal bupivacaine infiltration on the management of total knee arthroplasty. JAMA Surg. 2017; 152(1): 90-5.

12. Gibbins M, Kane C, Smit R, Rodseth R. Periarticular local anaesthetic in knee arthroplasty: a systematic review and meta-analysis of randomised trials. SA Orthop J. 2016; 15(3): 49-56.

13. Unver B, Yuksel E, Kalkan S, Maltepe F, Karatosun V. FRI0620-HPR efficacy of local infiltration analgesia in total knee arthroplasty: a randomised, placebo-controlled, double-blind trial. Ann Rheum Dis. 2016; 75(Suppl 2): 1278.1-8.

14. Hu B, Lin T, Yan SG, Tong SL, Yu JH, Xu JJ, et al. Local infiltration analgesia versus regional blockade for postoperative analgesia in total knee arthroplasty: a meta-analysis of randomized controlled trials. Pain Physician. 2016; 19(4): 205-14.

15. Barrington JW, Emerson RH, Lovald ST, Lombardi AV, Berend KR. No difference in early analgesia between liposomal bupivacaine injection and intrathecal morphine after TKA. Clin Orthop Relat Res. 2017; 475(1): 94-105.

16. Albrecht E, Guyen O, Jacot-Guillarmod A, Kirkham KR. The analgesic efficacy of local infiltration analgesia vs femoral nerve block after total knee arthroplasty: A systematic review and meta-analysis. Br J Anaesth. 2016; 116(5): 597-609.

17. Guild GN, Galindo RP, Marino J, Cushner FD, Scuderi GR. Periarticular regional analgesia in total knee arthroplasty. a review of the neuroanatomy and injection technique. Orthop Clin North Am [Internet]. 2015; 46(1): 1-8. Available from: http://dx.doi. org/10.1016/j.ocl.2014.09.016

18. Koh CK, Zeng I, Ravi S, Zhu M, Vince KG, Young SW. Periprosthetic joint infection is the main cause of failure for modern knee arthroplasty: an analysis of 11,134 knees. Clin Orthop Relat Res. 2017; 475(9): 2194-201.

19. Xing Q, Dai W, Zhao D, Wu J, Huang C, Zhao Y. Adductor canal block with local infiltrative analgesia compared with local infiltrate analgesia for pain control after total knee arthroplasty: a meta-analysis of randomized controlled trials. Med (Baltimore). 2017; 96(38): e8103. 